\title{
Commentary on "The Processing of Pitch and Scale: An ERP Study of Musicians Trained Outside of the Western Musical System" by Bischoff Renninger, Wilson, and Donchin
}

\author{
PETR JANATA \\ University of California, Davis [1]
}

\begin{abstract}
Electrophysiological measures of expectancy violation processing by the brain, such as the P300 component of the event-related potential, have provided insight into the way in which humans with varying amounts of musical experience maintain representations of musical information, in particular tonal representations. Bischoff Renninger and colleagues (2006) seek to extend this work by examining the P300 in the context of the very interesting topic of cross-cultural music perception, using Western listeners who either have or have not undergone training in Javanese music. Their study highlights the myriad issues and complexities of experimental design and analysis that must be addressed if one is to conduct an ethologically compelling and interpretable study of musical context representations using brain responses as dependent measures.
\end{abstract}

Received 2006 September 19; accepted 2006 September 21.

KEYWORDS: pitch, scale, ERP, enculturation

One of the outstanding questions in the field of music cognition is how listeners perceive the music of other cultures. This question arises quickly when one ponders whether our ability to understand and appreciate music is innate or learned, or when one contemplates statistical-learning models of music cognition (Huron, 2006; Tillmann, Bharucha, \& Bigand, 2000). Methods for measuring the brain's responses while listeners listen to and make judgments about music from different cultures hold promise of revealing what goes on in the brain's of individuals confronted with a musical system to which they have had little or no exposure. For example, in a recent study, Morrison and colleagues used functional magnetic resonance imaging (fMRI) to compare the perception of Western and Chinese speech and music in a group of musically-trained and untrained Western listeners (Morrison, Demorest, Aylward, Cramer, \& Maravilla, 2003). They found no differences in patterns of brain activations when listeners heard culturally foreign music, though they did find activation differences in prefrontal cortex as a function of musical training and the cultural origin of the music. While it is difficult to interpret null results, the failure to find clear evidence of a "foreign music detector," while observing effects that might be attributable to listening strategies, suggests that we may always need to consider mechanisms for assimilating culturally familiar and foreign music in the context of specific situational demands which may or may not have much to do with a realistic music listening experience.

The study by Bischoff Renninger and colleagues in this issue (Bischoff Renninger et al., 2006) utilizes a different measure of brain activity to look at the issue of cross-cultural music perception. The authors specifically focus on the P300 component of the event-related potential (ERP). A P300 is elicited by oddball stimuli, rare or surprising events that don't match an established context and therefore result in a process of "context updating" (Donchin \& Coles, 1988). Unexpected notes in melodies are no exception, as evidenced by the series of P300 studies cited by Bischoff-Renninger and colleagues. The authors reason that the P300 might be used to probe internalized representations of scales from different musical systems. For example, someone trained in Javanese music might readily detect and show a P300 response to a deviation from the pèlog scale, whereas a Western listener untrained in Javanese music would be oblivious to the deviation and therefore show no P300 (presuming the target event fit into the Western listener's schema).

Critical to the interpretation of P300 effects is a consideration of what constitutes the context. The P300 is famously an indicator of both global probability and local probability structures. On a global level, the lower the probability of any stimulus type in a stream of stimuli, e.g., a rare $800 \mathrm{~Hz}$ pitch embedded in a 
stream of $600 \mathrm{~Hz}$ pitches, the larger the P300 in response to the rare stimulus (Tueting, Sutton, \& Zubin, 1970). The amplitude of the P300 is shaped also at the local level, showing sensitivity to the probability structure of a sequence preceding a target event of interest (Squires, Wickens, Squires, \& Donchin, 1976). Given that perceived tonality is also shaped by both global and local melodic and harmonic probability structures (Oram \& Cuddy, 1995; Tillmann, Bigand, \& Pineau, 1998) or a combination of past and current experience with melodies written according to a particular tonal system (Kessler, Hansen, \& Shepard, 1984, as cited in Huron, 2006), those seeking to use the P300 to probe tonal knowledge must be especially mindful of the statistical properties of their stimulus, along with task characteristics, so as to not confound effects that are due to more stable internalized knowledge representations with the local statistical properties of the experimental stimuli.

Unfortunately, the current study by Bischoff Renninger and colleagues had several methodological confounds that preclude the drawing of any conclusions regarding the processing of scales from familiar and unfamiliar musical systems. The main confound is that membership in the set of infrequent stimuli was correlated with being a member of the non-diatonic set. Thus, two stimulus properties that are known to elicit a P300 response are entangled, and one can't be certain that the P300 effects that are observed are due to a violation of the tonal context instead of the global probability of the targets. The stimulus presentation parameters further complicated predicting what exactly any P300 responses might reflect: the stimuli were sequences of one hundred $350 \mathrm{~ms}$ sine tones, with $1600 \mathrm{~ms}$ separating the onsets of successive tones, hardly a compelling tonal context. Indeed, the authors mention that the experimental group had difficulty with the non-ethological nature of the sounds. The comparison of Western listeners with and without a modest amount of training in Javanese music was further clouded by the fact that the experimental group (those with training) showed larger ERPs overall than the control group, even for visual stimuli that should have presumably elicited comparable responses in the two groups. [Note: the legends in Figures 4 and 5 appear to be reversed for the standard and target pitches.]

The problems associated with the Bischoff Renninger et al. study are not insurmountable. Studies utilizing careful stimulus designs, in which diatonic and non-diatonic target tones are presented with realistic timbres and are matched in their frequency of occurrence within more realistic melodies, coupled with the testing of larger populations of subjects with extensive experience in the comparison tonal system, will shed much sought-after light on brain mechanisms underlying cross-cultural music perception.

\section{NOTES}

[1] Correspondence regarding this manuscript should be addressed to:

Petr Janata

Center for Mind and Brain

University of California, Davis

267 Cousteau Place

Davis, CA 95616

FAX: (530) 297-4400

phone: (530) 297-4471

e-mail: pjanata@ucdavis.edu

\section{REFERENCES}

Bischoff Renninger, L., Wilson, M., \& Donchin, E. (2006). The processing of pitch and scale: An ERP study of musicians trained outside the Western musical system. Empirical Musicology Review, Vol. 1, No. 4, pp. 185-197.

Donchin, E., \& Coles, M. G. H. (1988). Is the P300 component a manifestation of context updating? Behavioral and Brain Sciences, Vol. 11, No. 3, pp. 357-374. 
Huron, D. (2006). Sweet Anticipation: Music and the Psychology of Expectation. Cambridge, MA: MIT Press.

Kessler, E. J., Hansen, C., \& Shepard, R. N. (1984). Tonal schemata in the perception of music in Bali and in the West. Music Perception, Vol. 2, No. 2, pp. 131-165.

Morrison, S. J., Demorest, S. M., Aylward, E. H., Cramer, S. C., \& Maravilla, K. R. (2003). FMRI investigation of cross-cultural music comprehension. Neuroimage, Vol. 20, No. 1, pp. 378-384.

Oram, N., \& Cuddy, L. L. (1995). Responsiveness of Western adults to pitch-distributional information in melodic sequences. Psychological Research-Psychologische Forschung, Vol. 57, No. 2, pp. 103-118.

Squires, K. C., Wickens, C., Squires, N. K., \& Donchin, E. (1976). The effect of stimulus sequence on the waveform of the cortical event- related potential. Science, Vol. 193 (4258), pp. 1142-1146.

Tillmann, B., Bharucha, J. J., \& Bigand, E. (2000). Implicit learning of tonality: A self-organizing approach. Psychological Review, Vol. 107, No. 4, pp. 885-913.

Tillmann, B., Bigand, E., \& Pineau, M. (1998). Effects of global and local contexts on harmonic expectancy. Music Perception, Vol. 16, No. 1, pp. 99-117.

Tueting, P., Sutton, S., \& Zubin, J. (1970). Quantitative evoked potential correlates of the probability of events. Psychophysiology, Vol. 7, No. 3, pp. 385-394. 